\title{
Keratitis X Acremonium: A Case Report and Literature Review
}

\author{
Vanessa Cordeiro Dias ${ }^{1}$, Robert Gomes Cotta ${ }^{1}$, Ricardo Villela Bastos ${ }^{2}$, \\ Lucas Quinet de Andrade Bastos ${ }^{1}$, Victor Quinet de Andrade Bastos ${ }^{2}$ and \\ André Netto Bastos ${ }^{1}$ \\ ${ }^{1}$ Federal University of Juiz de Fora, Juiz de Fora, MG, Brazil \\ ${ }^{2}$ Cortes Villela Laboratory, Juiz de Fora, MG, Brazil
}

\section{Case Report \\ Volume 6 Issue 1}

Received Date: March 09, 2021

Published Date: March 23, 2021

DOI: $10.23880 /$ oajmb-16000187

*Corresponding author: Vanessa Cordeiro Dias, Federal University of Juiz de Fora-MG, Rua José Lourenço Kelmer, s/n - University Campus Bairro São Pedro, Juiz de Fora-MG, Brazil, Tel: +553221023213, Email: vancdias2@hotmail.com

\section{Abstract}

Purpose: Describe an unusual development of fungal keratitis caused by Acremonium sp in six patients who underwent cataract surgery at an ophthalmology service in Brazil, as well as to report the origin of these infections. Methods: Swabs from the affected corneas were collected to perform culture for bacteria and fungi. These materials were sown in Blood agar (Difco/ USA), Macconkey agar (Difco/USA) and Thioglycolate broth (Difco/USA) for bacterial research and Sabouraud agar (Difco/ USA) for fungi research. A microbiological study was carried out to analyze surgical instruments, the environment and other materials used in the surgeries. Results: Case reports occurred with six patients, all aged over 71 and 85 years, who underwent a surgical procedure for cataract correction by an Ophthalmology service. After surgery, these patients presented loss of unilateral visual capacity, with the formation of a white mass on the cornea. Swabs from the affected corneas were collected to perform culture for bacteria and fungi, in specific culture media. The bacterial cultures showed negative results. Fungal cultures revealed the presence of Acremonium spp. Conclusions: It is likely that Acremonium spores found in the wardrobe were deposited on all the sterile material stored there. At the time of cataract surgeries, this material was unpacked and fungal structures became detached and found in the surgical field of these patients' eyes a gateway. Thus, cleaning and sanitizing measures for surfaces must be implemented and monitored, especially in critical areas such as in hospital areas, in order to avoid damage to patients' health.

Keywords: Keratitis; Acremonium;Cataract Surgeries; Fungal

\section{Introduction}

An inflammation of the cornea secondary to infection is called keratitis infectious, which can be caused by viral, bacterial, parasitic or fungal agents [1]. Infectious keratitis, ocular trauma, post-surgical bullous keratopathy and corneal degenerations are the major risk factors that contribute to blindness. Fungal keratitis is an important cause of ocular damages and blindness especially in regions of warm and humid climate [2]. In immunocompetent individuals keratitis and endophthalmitis are frequently associated with ocular trauma [3]. Traditionally, yeasts were the main pathogen of fungal keratitis, while now filamentous represents the majority of the cases, which represents a change in the causative pathogens and risk factors [4]. Acremonium $s p$ are filamentous saprophytic fungi commonly isolated from the environment, frequently found in soil and dead plants [3]. Candida, Fusarium, and Aspergillus species are the most 


\section{Open Access Journal of Microbiology \& Biotechnology}

common fungal keratitis agents, while Acremonium is a rare etiological agent compared with another fungal keratitis [4]. However, it is associated with serious consequences for the patient that can lead to corneal morbidity and even blindness [5].

In ophthalmology practice, fungal keratitis is more difficult to diagnose and treat. The microbiological investigations contribute to the correct identification of the agent and assists in the establishment of therapeutic approaches [1]. In general, to treat fungal keratitis, topical natamycin is the first line of treatment, although amphotericin B could be suggested too [5]. Furthermore, corneal disease represents the second cause of blindness worldwide and cornea transplantation is, currently, a successful and helpful treatment method [6]. However, although uncommon, infectious keratitis is a serious complication after keratoplasty. Fungal are the second most common agent while bacterial keratitis constitute the majority of the cases [7]. On the other hand, in developing countries, corneal complications due to cataract surgery has increasing the prevalence of cornea blindness [2]. Thus, the possibility of traumatic fungal inoculation after keratoplasty and cataract surgery must be carefully evaluated, considering that late diagnosis and inadequate therapy may be associated with serious damages to the patient. The aim of this study is to describe an unusual development of fungal keratitis caused by Acremonium $s p$ in six patients who underwent cataract surgery at an ophthalmology service in Brazil, as well as to report the origin of these infections.

\section{Methods}

Swabs from the affected corneas were collected to perform culture for bacteria and fungi. These materials were sown in Blood agar (Difco/USA), Macconkey agar (Difco/ USA) and Thioglycolate broth (Difco/USA) for bacterial research and Sabouraud agar (Difco/USA) for fungi research. In view of the sequence of cases of patients infected with Acremonium spp, a microbiological study was carried out to analyze surgical instruments, the environment and other materials used in the surgeries (Table 1). With the aid of a swab soaked in sterile saline solution, it was possible to perform microbiological analysis of non-biological materials in Sabouraud agar (Difco/USA) for fungi research.

\begin{tabular}{|c|c|c|}
\hline Cirurgical instruments & Environment & Others \\
\hline Wardrobe & Detergent & Autoclave \\
\hline Needle & Swab & Carrying Case \\
\hline Pipe & Hospital Bed & Autoclave Steam \\
\hline Material Box & Air Conditioning & \\
\hline Injector & Wall & \\
\hline Injectors Box & & \\
\hline Handles & & \\
\hline Tweezers & & \\
\hline Scissors & & \\
\hline Extra Capsular Box & & \\
\hline Cannulas & & \\
\hline Cannula Box & & \\
\hline Spatula & & \\
\hline Claws & & \\
\hline
\end{tabular}

Table 1: Non-biological materials submitted to microbiological analysis.

\section{Case Report}

Case reports occurred with six patients, all aged over 71 and 85 years, who underwent a surgical procedure for cataract correction by an Ophthalmology service (Table 2).
After surgery, these patients presented loss of unilateral visual capacity, with the formation of a white mass on the cornea. For medical advice, these patients were referred to a clinical laboratory for a microbiological study, aiming at the identification of a possible microorganism, which would 


\section{Open Access Journal of Microbiology \& Biotechnology}

justify the occurrence of blindness. Swabs from the affected corneas were collected to perform culture for bacteria and fungi. These materials were sown in specific culture media. The bacterial cultures showed negative results. Fungal cultures revealed the presence of Acremonium spp (Figure 1).

\begin{tabular}{|c|c|c|}
\hline Cases (n=6) & Age (Years) & Gender (M/F) \\
\hline Patient 1 & 82 & M \\
\hline Patient 2 & 85 & F \\
\hline Patient 3 & 79 & F \\
\hline Patient 4 & 82 & M \\
\hline Patient 5 & 71 & F \\
\hline Patient 6 & 82 & M \\
\hline
\end{tabular}

Table 2: Demographic Data of Fungal Keratitis Patients.

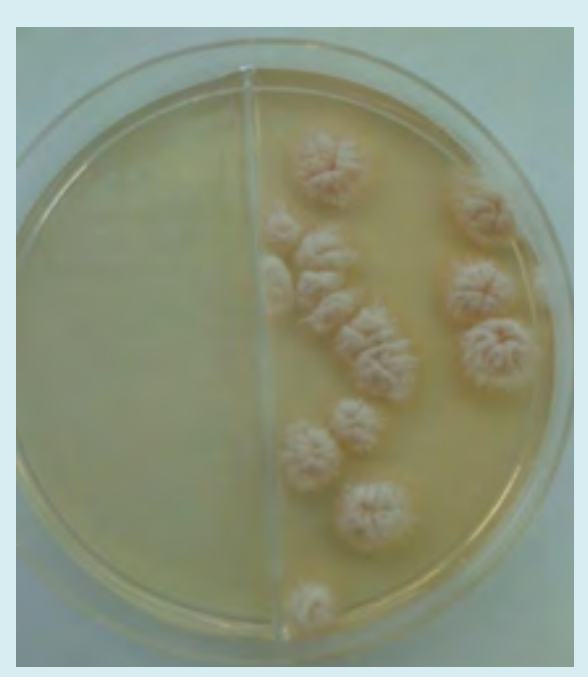

Figure 1: Colony formation on Sabouraud agar exhibited a white, creamy and fluffy appearance.

The surgeries were performed by the same team (doctor and instructor) on different dates. The surgical procedure of all patients was performed using disposable and sterile materials and other non-disposable materials, but sterilized by the Ophthalmology service. All disposable and sterile materials were within the expiration date recommended by the manufacturers and were stored according to instructions. The sterilization process of non-disposable materials, performed by the Ophthalmology service, proved to be effective. All sterilization equipment has previously undergone preventive maintenance. All professionals working in the sterilization of materials had received training prior to the start of their activities. In view of the sequence of cases of patients infected with Acremonium spp, a microbiological study was carried out to analyze surgical instruments, the environment and other materials used in the surgeries (Table 1). With the aid of a swab soaked in sterile saline solution, it was possible to perform microbiological analysis of non-biological materials. These analyzes showed that the sample collected from the interior of a wardrobe where sterile surgical instruments were stored had Acremonium spp. The others analyzed materials showed negative results for fungus. Patients underwent treatment with local corticosteroids and amphotericin b, with no therapeutic success. All patients evolved with $100 \%$ loss of visual acuity in the eye submitted to the surgical procedure.

\section{Discussion}

Filamentous fungi, such as Acremonium species (A. falciforme, A. kiliensis and A. recifei) are common environmental saprophytes that cause a variety of infections, mainly considered secondary to colonization and increased host susceptibility [7-9]. Thus, trauma to the cornea by aggression with plant material is the main risk factor for the development of fungal keratitis in tropical regions or developing countries [7]. However, our research, realized in Brazil, showed injury to the cornea as a result of trauma caused by surgical instruments contaminated with Acremonium. In the present study, all six patients of advanced age, considered immunocompetent, had a history of cataract surgery. It is likely that Acremonium spores found in the wardrobe were deposited on all the sterile material stored there. At the time of cataract surgeries, this material was unpacked and fungal structures became detached and found in the surgical field of these patients' eyes a gateway. Then, because Acremonium species are cosmopolitan in nature, they can also be encountered as contaminants in several areas, such as the wardrobe in this hospital $[9,10]$.

Thus, cleaning and sanitizing measures for surfaces must be implemented and monitored, especially in critical areas such as in hospital areas, in order to avoid damage to patients' health.

\section{References}

1. Satpathy G, Ahmed NH, Nayak N, Tandon R, Sharma N, et al. (2019) Spectrum of mycotic keratitis in north India: Sixteen years study from a tertiary care ophthalmic centre. J Infect Public Health 12(3): 367-371.

2. Gupta N, Vashist P, Tandon R, Gupta SK, Dwivedi S, et al. (2015) Prevalence of corneal diseases in the rural Indian population: the Corneal Opacity Rural Epidemiological (CORE) study. Br J Ophthalmol 99(2):147-152.

3. Loakimidou A, Vyzantiadis TA, Sakellari I, Arabatzis M, Smias C, et al. (2013) An unusual cluster of Acremonium kiliense fungaemias in a haematopoietic cell 


\section{Open Access Journal of Microbiology \& Biotechnology}

transplantation unit. Diagn Microbiol Infect Dis 75(3): 313-316.

4. Jurkunas U, Behlau I, Colby K (2009) Fungal keratitis: changing pathogens and risk factors. Cornea 28(6): 638643.

5. Yagci A, Palamar M, Polat Hilmioglu S, Irkec M (2016) Cross-Linking Treatment and Corneal Transplant in Refractory Acremonium Keratitis: Case Report. Exp Clin Transplant 14(5): 580-583.

6. Li GG, Zhu H, Ji CN, Zang XJ (2019) Microbiological contamination in donor corneas preserved for mediumterm. Cell Tissue Bank 20(3): 379-387.

7. Seong-Jae K, Yong-Wun C, Seong-Wook S, Sun-Joo K, Ji-
Myong Y (2014) Clinical experiences in fungal keratitis caused by Acremonium. Clin Ophthalmol (8): 283-287.

8. Fincher RM, Fisher JF, Lovell RD, Newman CL, EspinelIngroff A, et al. (1991) Infection due to the fungus Acremonium (cephalosporium). Medicine (Baltimore) 70(6): 398-409.

9. Verghese $S$ (2010) Post traumatic fungal keratitis caused by Acremonium recifei. Indian J Pathol Microbiol 53(3): 587-588.

10. Mahmoudi S, Masoomi A, Ahmadikia K, Tabatabaei SA, Soleimani M, et al. (2018) Fungal keratitis: An overview of clinical and laboratory aspects. Mycoses 61(12): 916930. 